Received: 31 August 2017

Accepted: 27 November 2017

Published online: 08 December 2017

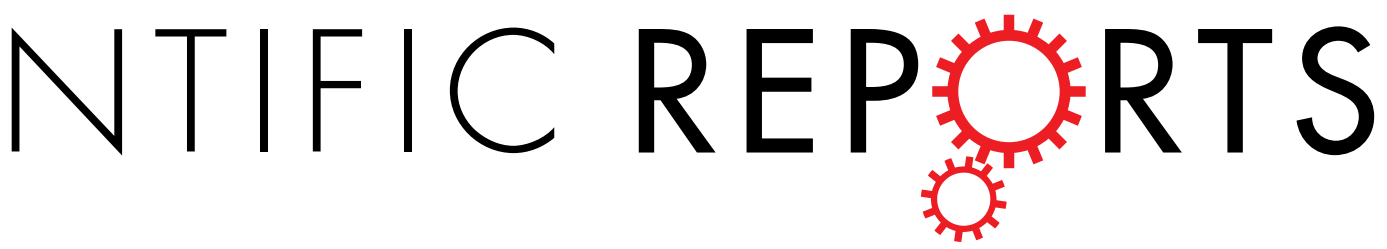

\title{
Detection and measure of genuine tripartite entanglement with partial transposition and realignment of density matrices
}

\author{
Ming $\mathrm{Li}^{1}$, Jing Wang ${ }^{1}$, Shuqian Shen ${ }^{1}$, Zhihua Chen ${ }^{2}$ \& Shao-Ming Fei, ${ }^{3,4}$
}

It is challenging task to detect and measure genuine multipartite entanglement. We investigate the problem by considering the average based positive partial transposition(PPT) criterion and the realignment criterion. Sufficient conditions for detecting genuine tripartite entanglement are presented. We also derive lower bounds for the genuine tripartite entanglement concurrence with respect to the conditions. While the PPT criterion and the realignment criterion are powerful for detecting bipartite entanglement and for providing lower bounds of bipartite concurrences, our results give an effective operational way to detect and measure the genuine tripartite entanglement.

Quantum entanglement is recognized as a remarkable resource in the rapidly expanding field of quantum information science, with various applications ${ }^{1}$. A multipartite quantum state that is not separable with respect to any bi-partition is said to be genuinely multipartite entangled $(\mathrm{GME})^{2}$, which is one of the important type of entanglement, and offers significant advantage in quantum tasks comparing with bipartite entanglement ${ }^{3}$. In particular, it is the basic ingredient in measurement-based quantum computation ${ }^{4}$, and is beneficial in various quantum communication protocols, including secret sharing ${ }^{5,6}$, extreme spin squeezing ${ }^{7}$, high sensitivity in some general metrology tasks ${ }^{8}$, quantum computing using cluster states ${ }^{9}$, and multiparty quantum network ${ }^{10}$. Although its significance, detecting and measuring such kind of entanglement turns out to be quite difficult. To certify GME, an abundance of linear and nonlinear entanglement witnesses ${ }^{11-19}$, generalized concurrence for multi genuine entanglement ${ }^{20-23}$, and Bell-like inequalities ${ }^{24} e^{-n}$ tanglement witnesses were derived (see e.g. reviews ${ }^{2,25}$ ) and a characterisation in terms of semi-definite programs (SDP) was developed ${ }^{26,27}$. Nevertheless, the problem remains far from being satisfactorily solved.

For bipartite systems, Peres in $^{28}$ has presented a much stronger separability criterion, which is called positive partial transpose (PPT) criterion. It says that if $\rho_{A B}$ is separable, then the partial transposition $\rho_{A B}^{T_{B}}$ with matrix elements defined as: $\left(\rho_{A B}^{T_{B}}\right)_{i j, k l}=\rho_{i l, k j}$ is a density operator (i.e. has nonnegative spectrum). It has interpretation as a partial time reversal ${ }^{29}$. There is yet another strong class of criteria based on linear contractions on product states. They stem from the new criterion discovered in ${ }^{30,31}$ called computable cross norm criterion or matrix realignment criterion(CCNR) which is operational and independent on PPT test ${ }^{28}$. In terms of matrix elements it can be stated as follows: if the state $\rho_{A B}$ is separable then the matrix $\mathcal{R}(\rho)$ with elements $\mathcal{R}(\rho)_{i j, k l}=\rho_{i k, j l}$ has trace norm not greater than one, i.e. $\|\mathcal{R}(\rho)\|_{K F} \leq 1$. Quite remarkably, the realignment criterion has been found to be able to detect some PPT entangled states ${ }^{30,31}$ and to be useful for construction of some nondecomposable maps. It also provides nice lower bound on concurrence ${ }^{32}$. Further more, a necessary and sufficient criterion of the local unitary equivalence for general multipartite states based on matrix realignment has been presented in $^{33}$.

In this manuscript, we investigate the detection of GME for arbitrary tripartite quantum systems. We will derive an effective criterion based on PPT and CCNR. A lower bound for GME concurrence will be also obtained. We then compute examples to show the effectiveness of our results.

${ }^{1}$ College of the Science, China University of Petroleum, Qingdao, 266580, P. R. China. ${ }^{2}$ Department of Science, Zhijiang college, Zhejiang University of Technology, Hangzhou, 310024, P. R. China. ${ }^{3}$ School of Mathematical Sciences, Capital Normal University, Beijing, 100048, P. R. China. ${ }^{4}$ Max-Planck-Institute for Mathematics in the Sciences, Leipzig, 04103, Germany. Correspondence and requests for materials should be addressed to J.W. (email: alabaila@163.com) 


\section{Results}

In the following, we present a criterion to detect GME for tripartite qudits systems by using the PPT and CCNR criteria. A lower bound for GME concurrence of tripartite quantum systems will be also obtained. We start with some definitions and notations.

Let $H_{i}^{d}, i=1,2,3$, denote $d$-dimensional Hilbert spaces. A tripartite state $\rho \in H_{1}^{d} \otimes H_{2}^{d} \otimes H_{3}^{d}$ can be expressed as $\rho=\sum p_{\alpha}\left|\psi_{\alpha}\right\rangle\left\langle\psi_{\alpha}\right|$, where $0<p_{\alpha} \leq 1, \sum p_{\alpha}=1,\left|\psi_{\alpha}\right\rangle \in H_{1}^{d} \otimes H_{2}^{d} \otimes H_{3}^{d}$ are normalized pure states. If all $\left|\psi_{\alpha}\right\rangle$ are biseparable, namely, either $\left|\psi_{\alpha}\right\rangle=\left|\varphi_{\alpha}^{1}\right\rangle \otimes\left|\varphi_{\alpha}^{23}\right\rangle$ or $\left|\psi_{\beta}\right\rangle=\left|\varphi_{\beta}^{2}\right\rangle \otimes\left|\varphi_{\beta}^{13}\right\rangle$ or $\left|\psi_{\gamma}\right\rangle=\left|\varphi_{\gamma}^{3}\right\rangle \otimes\left|\varphi_{\gamma}^{12}\right\rangle$, where $\left|\varphi_{\gamma}^{i}\right\rangle$ and $\left|\varphi_{\gamma}^{i j}\right\rangle$ denote pure states in $H_{i}^{d}$ and $H_{i}^{d} \otimes H_{j}^{d}$ respectively, then $\rho$ is said to be bipartite separable. Otherwise, $\rho$ is called genuine multipartite entangled.

Define that $M(\rho)=\frac{1}{3}\left(\left\|\rho^{T_{1}}\right\|+\left\|\rho^{T_{2}}\right\|+\left\|\rho^{T_{3}}\right\|\right), N(\rho)=\frac{1}{3}\left(\left\|R_{1 \mid 23}(\rho)\right\|+\left\|R_{2 \mid 13}(\rho)\right\|+\left\|R_{3 \mid 12}(\rho)\right\|\right)$, where $T_{i}$ s are the partial transposition over the $i$ th subsystem, $i=1,2,3$ and $R_{i \mid j k}$ stands for the bipartite realignment with respect to subsystem $i$ and subsystems $j k, i, j, k=1,2,3 .\|\cdot\|$ denotes the trace norm of a matrix.

To derive GME criterion, we first obtain the following lemma.

Lemma: Let $d=\min \{m, n\}$. For a bipartite quantum state $|\varphi\rangle \in H_{A}^{m} \otimes H_{B}^{n}$, we have $\left\|(|\varphi\rangle\langle\varphi|)^{T_{A}}\right\| \leq d$, and $\left\|R_{A \mid B}(|\varphi\rangle\langle\varphi|)\right\| \leq d$.

Proof. By Schmidt decomposition, we set $|\varphi\rangle=\sum_{i=1}^{d} \sqrt{u_{i}}|i i\rangle$ with $\sum_{i=1}^{d} u_{i}=1, u_{i} \geq 0$. By the Cauchy-Schwarz inequality one computes

$$
\left\|(|\varphi\rangle\langle\varphi|)^{T_{A}}\right\|=\left\|R_{A \mid B}(|\varphi\rangle\langle\varphi|)\right\|=\left(\sum_{i} \sqrt{u_{i}}\right)^{2} \leq d\left(\sum_{i} u_{i}\right)^{2}=d .
$$

Then we are ready to show the theorems.

Theorem 1: Let $\rho \in H_{123}=H_{1}^{d} \otimes H_{2}^{d} \otimes H_{3}^{d}$ be a tripartite qudits quantum state. If $\rho$ is bipartite separable, then $\max \{M(\rho), N(\rho)\} \leq \frac{1+2 d}{3}$ must hold. Or equivalently, if $\max \{M(\rho), N(\rho)\}>\frac{1+2 d}{3}$, then $\rho$ is GME.

See Methods for the proof of theorem 1.

The GME concurrence for tripartite quantum systems, which is defined as follows, is proved to be a well defined measure ${ }^{20,21}$. For a pure state $|\psi\rangle \in H_{1}^{d} \otimes H_{2}^{d} \otimes H_{3}^{d}$, the GME concurrence is defined by

$$
C_{G M E}(|\psi\rangle)=\sqrt{\min \left\{1-\operatorname{tr}\left(\rho_{1}^{2}\right), 1-\operatorname{tr}\left(\rho_{2}^{2}\right), 1-\operatorname{tr}\left(\rho_{3}^{2}\right)\right\}},
$$

where $\rho_{i}$ is the reduced matrix for the $i$ th subsystem. For mixed state $\rho \in H_{1}^{d} \otimes H_{2}^{d} \otimes H_{3}^{d}$, the GME concurrence is then defined by the convex roof

$$
C_{G M E}(\rho)=\min \sum_{\left\{p_{\alpha},\left|\psi_{\alpha}\right\rangle\right\}} p_{\alpha} C_{G M E}\left(\left|\psi_{\alpha}\right\rangle\right)
$$

The minimum is taken over all pure ensemble decompositions of $\rho$. Since one has to find the optimal ensemble to do the minimization, the GME concurrence is hard to compute. In the following we derive an effective lower bound for GME concurrence in terms of the PPT criterion and the CCNR criterion.

Theorem 2: Let $\rho \in H_{123}=H_{1}^{d} \otimes H_{2}^{d} \otimes H_{3}^{d}$ be a tripartite qudits quantum state. Then one has

$$
C_{G M E}(\rho) \geq \frac{1}{\sqrt{d(d-1)}}\left(\max \{M(\rho), N(\rho)\}-\frac{1+2 d}{3}\right) .
$$

See Methods for the proof of theorem 2.

Applications. The following two examples show that the criterion and the lower bound of GME concurrence above are much effective for detecting and measuring GME in tripartite quantum systems.

Example 1: Consider quantum state $\rho \in H_{1}^{3} \otimes H_{2}^{3} \otimes H_{3}^{3}, \rho=\frac{1-x}{27} I+x|\varphi\rangle\langle\varphi|$, where $|\varphi\rangle=\frac{1}{\sqrt{3}}(|000\rangle+$ $|111\rangle+|222\rangle)$ is the GHZ state. By Theorem 1 in $^{13}$ we can detect GME for $0.894427<x \leq 1$. Using the Theorem 1 in this manuscript, we compute $\max \{M(\rho), N(\rho)\}=\frac{1}{9}(8+10 x+|10 x-1|)$. Thus GME is detected for $0.7<x \leq 1$.

Example 2: We consider the mixture of the GHZ state and W state in three-qubit quantum systems $\rho=\frac{1-x-y}{8} I+x|G H Z\rangle\langle G H Z|+y| W\rangle\langle W|$, where $|G H Z\rangle=\frac{1}{\sqrt{2}}(|000\rangle+|111\rangle)$ and $|W\rangle=\frac{1}{\sqrt{3}}(|001\rangle+$ $|010\rangle+|100\rangle)$. As shown in Fig. 1, our criterion detect some GME(blue region) that can not be detected by Vicente criteria.

The lower bound of GME concurrence in Theorem 2 for $\rho$ is computed to be 


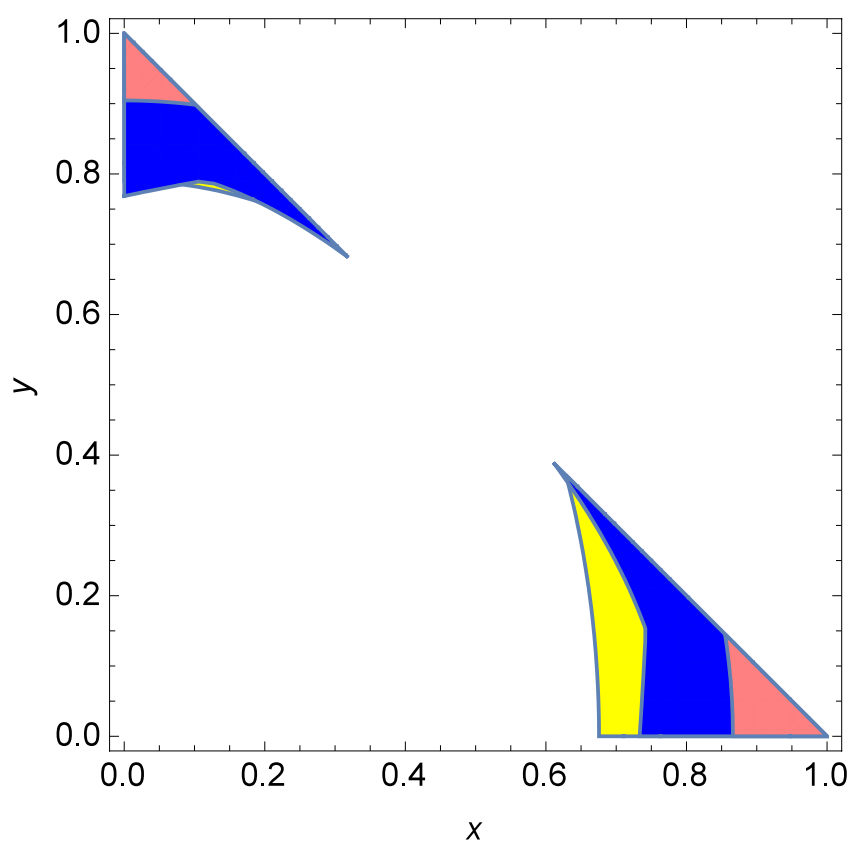

Figure 1. GME Detected by Vicente criterion (pink region by Theorem 1 and yellow region by Theorem 2 in $^{13}$ ) and by the theorem 1 in this manuscript(blue region).

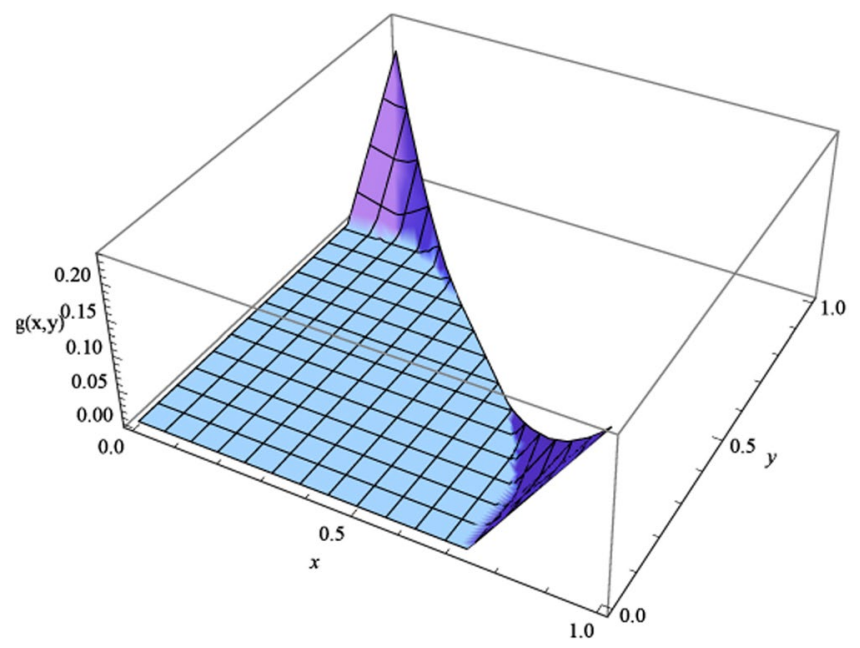

Figure 2. The lower bound of GME concurrence for $\rho$ in example 2. $g(x, y)$ stands for the lower bound.

$$
\begin{aligned}
g(x, y)= & (1 /(24 \sqrt{2}))\left(-40+3 \sqrt{(-1-3 \alpha+\beta)^{2}}+6 \sqrt{(-1+\alpha+\beta)^{2}}+\sqrt{(3-3 \alpha+13 \beta)^{2}}\right. \\
& +\sqrt{9+153 \alpha^{2}+6 \beta+17 \beta^{2}-6 \alpha(3+\beta)-8 \sqrt{(3-3 \alpha+\beta)^{2}\left(9 \alpha^{2}+\beta^{2}\right)}} \\
& +\sqrt{9+153 \alpha^{2}+6 \beta+17 \beta^{2}-6 \alpha(3+\beta)+8 \sqrt{(3-3 \alpha+\beta)^{2}\left(9 \alpha^{2}+\beta^{2}\right)}} \\
& +\sqrt{9+45 \alpha^{2}-18 \alpha(-1+\beta)-18 \beta+137 \beta^{2}-12 \sqrt{(1+\alpha-\beta)^{2}\left(9 \alpha^{2}+32 \beta^{2}\right)}} \\
& +\sqrt{\left.9+45 \alpha^{2}-18 \alpha(-1+\beta)-18 \beta+137 \beta^{2}+12 \sqrt{(1+\alpha-\beta)^{2}\left(9 \alpha^{2}+32 \beta^{2}\right)}\right)}
\end{aligned}
$$

as ploted in Fig. 2.

\section{Discussions}

It is a basic and fundamental question in quantum information theory to detect and measure GME. In this manuscript we have presented a GME criterion based on the PPT and Realignment criteria. A lower bound of GME concurrence for tripartite quantum system has also been obtained. Examples show that our criterion is 
independent of Vicente criteria and can detect more genuine entangled quantum states. Our results are derived by average based PPT and CCNR criteria. One can construct more effective criteria to detect GME and lower bounds of GME concurrence by taking the average of the correlation matrices or covariance matrices and so on. It is also of interesting to investigate the implementation of the criterion with measurements or to extend the results to systems consisting of more than three parties.

\section{Methods}

Proof of Theorem 1 Lets consider a pure state $\rho=|\psi\rangle\langle\psi|$ first. Assume that $|\psi\rangle \in H_{123}=H_{1}^{d} \otimes H_{2}^{d} \otimes H_{3}^{d}$ be bipartite separable, which will be in one of the following three forms: $|\psi\rangle=\left|\varphi_{1}\right\rangle \otimes\left|\varphi_{23}\right\rangle,|\psi\rangle=\left|\varphi_{2}\right\rangle \otimes\left|\varphi_{13}\right\rangle$, or $|\psi\rangle=\left|\varphi_{3}\right\rangle \otimes\left|\varphi_{12}\right\rangle$. If $|\psi\rangle=\left|\varphi_{1}\right\rangle \otimes\left|\varphi_{23}\right\rangle$, then by using the first two equations in (1) we have

$$
\begin{aligned}
& \left\|\rho^{T_{1}}\right\|=\left\|R_{1 \mid 23}(\rho)\right\|=\|\left(\left|\varphi_{1}\right\rangle\left\langle\varphi_{1}\right|\right)^{T_{1}} \otimes\left|\varphi_{23}\right\rangle\left\langle\varphi_{23}\right| \|=1 ; \\
& \left\|\rho^{T_{2}}\right\|=\left\|R_{2 \mid 13}(\rho)\right\|=\|\left|\varphi_{1}\right\rangle\left\langle\varphi_{1}\right|\|\cdot\|\left(\left|\varphi_{23}\right\rangle\left\langle\varphi_{23}\right|\right)^{T_{2}}\|=\|\left(\left|\varphi_{23}\right\rangle\left\langle\varphi_{23}\right|\right)^{T_{2}} \| \leq d ; \\
& \left\|\rho^{T_{3}}\right\|=\left\|R_{3 \mid 12}(\rho)\right\|=\|\left|\varphi_{1}\right\rangle\left\langle\varphi_{1}\right|\|\cdot\|\left(\left|\varphi_{23}\right\rangle\left\langle\varphi_{23}\right|\right)^{T_{2}} \| \leq d .
\end{aligned}
$$

Similarly, one has

$$
\left\|\rho^{T_{1}}\right\|=\left\|R_{1 \mid 23}(\rho)\right\| \leq d ;\left\|\rho^{T_{2}}\right\|=\left\|R_{2 \mid 13}(\rho)\right\|=1 ;\left\|\rho^{T_{3}}\right\|=\left\|R_{3 \mid 12}(\rho)\right\| \leq d
$$

for $|\psi\rangle=\left|\varphi_{2}\right\rangle\left\langle\varphi_{13}\right|$ and

$$
\left\|\rho^{T_{1}}\right\|=\left\|R_{1 \mid 23}(\rho)\right\| \leq d ;\left\|\rho^{T_{2}}\right\|=\left\|R_{2 \mid 13}(\rho)\right\| \leq d ;\left\|\rho^{T_{3}}\right\|=\left\|R_{3 \mid 12}(\rho)\right\|=1
$$

for $|\psi\rangle=\left|\varphi_{3}\right\rangle\left\langle\varphi_{12}\right|$ respectively. Thus for any type bipartite separable pure quantum state, we always have $M(\rho) \leq \frac{1+2 d}{3}$, and $N(\rho) \leq \frac{1+2 d}{3}$.

For mixed bipartite separable state $\rho$, by using the convex property of $M(\rho)$ and $N(\rho)$ we obtain

$$
M(\rho) \leq \sum p_{\alpha} M\left(\left|\psi_{\alpha}\right\rangle\left\langle\psi_{\alpha}\right|\right) \leq \frac{1+2 d}{3},
$$

and

$$
N(\rho) \leq \sum p_{\alpha} N\left(\left|\psi_{\alpha}\right\rangle\left\langle\psi_{\alpha}\right|\right) \leq \frac{1+2 d}{3},
$$

which proves the theorem.

Proof of Theorem 2

Still we consider a pure state first. Let $\rho=|\psi\rangle\langle\psi| \in H_{1}^{d} \otimes H_{2}^{d} \otimes H_{3}^{d}$ be a pure quantum state. From the result in $^{32}$, we have

$$
\begin{aligned}
& \sqrt{1-\operatorname{tr} \rho_{1}^{2}} \geq \frac{1}{\sqrt{d(d-1)}}\left(\left\|\rho^{T_{1}}\right\|-1\right) \\
& \sqrt{1-\operatorname{tr} \rho_{2}^{2}} \geq \frac{1}{\sqrt{d(d-1)}}\left(\| \rho^{T_{2}}||-1\right) \\
& \sqrt{1-\operatorname{tr} \rho_{3}^{2}} \geq \frac{1}{\sqrt{d(d-1)}}\left(\left\|\rho^{T_{3}}\right\|-1\right) .
\end{aligned}
$$

One computes

$$
\begin{aligned}
& 3 \sqrt{d(d-1)} \sqrt{1-\operatorname{tr} \rho_{1}^{2}}-3 \max \{M(\rho), N(\rho)\}+1+2 d \\
= & 3 \sqrt{d(d-1)} \sqrt{1-\operatorname{tr} \rho_{1}^{2}}-\left(\left\|\rho^{T_{1}}\right\|+\left\|\rho^{T_{2}}\right\|+\left\|\rho^{T_{3}}\right\|\right)+1+2 d \\
\geq & 3 \sqrt{d(d-1)} \sqrt{1-\operatorname{tr} \rho_{1}^{2}}-\sqrt{d(d-1)}\left(\sqrt{1-\operatorname{tr} \rho_{1}^{2}}+\sqrt{1-\operatorname{tr} \rho_{2}^{2}}+\sqrt{1-\operatorname{tr} \rho_{3}^{2}}\right)-2+2 d \\
= & 2 \sqrt{d(d-1)} \sqrt{1-\operatorname{tr} \rho_{1}^{2}}-\sqrt{d(d-1)}\left(\sqrt{1-\operatorname{tr} \rho_{2}^{2}}+\sqrt{1-\operatorname{tr} \rho_{3}^{2}}\right)-2+2 d \\
\geq & 2 \sqrt{d(d-1)} \sqrt{\frac{d-1}{d}}-2+2 d=0,
\end{aligned}
$$

where we have used $\sqrt{1-\operatorname{tr} \rho_{1}^{2}} \geq 0$ and $\sqrt{1-\operatorname{tr} \rho_{k}^{2}} \leq 1-\frac{1}{d}, k=2$ or 3 to obtain the last inequality above.

Thus we get 


$$
\sqrt{1-\operatorname{tr} \rho_{1}^{2}} \geq \frac{1}{\sqrt{d(d-1)}}\left(\max \{M(\rho), N(\rho)\}-\frac{1+2 d}{3}\right) .
$$

Similarly we obtain

$$
\begin{aligned}
& \sqrt{1-\operatorname{tr} \rho_{2}^{2}} \geq \frac{1}{\sqrt{d(d-1)}}\left(\max \{M(\rho), N(\rho)\}-\frac{1+2 d}{3}\right) . \\
& \sqrt{1-\operatorname{tr} \rho_{3}^{2}} \geq \frac{1}{\sqrt{d(d-1)}}\left(\max \{M(\rho), N(\rho)\}-\frac{1+2 d}{3}\right) .
\end{aligned}
$$

Then according to the definition of GME concurrence, we derive

$$
C_{G M E}(|\psi\rangle) \geq \frac{1}{\sqrt{d(d-1)}}\left(\max \{M(\rho), N(\rho)\}-\frac{1+2 d}{3}\right) .
$$

Now we consider a mixed state $\rho \in H_{1}^{d} \otimes H_{2}^{d} \otimes H_{3}^{d}$ with the optimal ensemble decomposition $\rho=\sum_{\alpha} p_{\alpha}\left|\psi_{\alpha}\right\rangle$ $\left\langle\psi_{\alpha}\right|$ s.t. the GME concurrence attains its minimum. One gets

$$
\begin{aligned}
C_{G M E}(\rho) & =\sum_{p_{\alpha},\left|\psi_{\alpha}\right\rangle} p_{\alpha} C_{G M E}\left(\left|\psi_{\alpha}\right\rangle\right) \\
& \geq \frac{1}{\sqrt{d(d-1)}}\left(\max \{M(\rho), N(\rho)\}-\frac{1+2 d}{3}\right) \sum_{\alpha} p_{\alpha} \\
& =\frac{1}{\sqrt{d(d-1)}}\left(\max \{M(\rho), N(\rho)\}-\frac{1+2 d}{3}\right)
\end{aligned}
$$

which ends the proof of the theorem.

\section{References}

1. Nielsen, M. A. \& Chuang, I. L. Quantum Computation and Quantum Information (Cambridge University Press, Cambridge, England, 2000)

2. Gühne, O. \& Tóth, G. Entanglement detection. Phys. Rep. 474, 1-75 (2009).

3. Horodecki, R., Horodecki, P., Horodecki, M. \& Horodecki, K. Quantum entanglement. Rev. Mod. Phys. 81, 865 (2009).

4. Briegel, H. J., Browne, D. E., Dür, W., Raussendorf, R. \& Van den Nest, M. Measurement-based quantum computation. Nat. Phys. 5, 19 (2009).

5. Gisin, N., Ribordy, G., Tittel, W. \& Zbinden, H. Quantum cryptography. Rev. Mod. Phy. 74, 145 (2002).

6. Hillery, M., Bužek, V. \& Berthiaume, A. Quantum secret sharing. Phys. Rev. A 59, 1829 (1999).

7. Srensen, A. S. \& Mlmer, K. Entanglement and Extreme Spin Squeezing. Phys. Rev. Lett. 86, 4431 (2001).

8. Tóth, G. Multipartite entanglement and high-precision metrology. Phys. Rev. A 85, 022322 (2012).

9. Raussendorf, R. \& Briegel, H. J. A One-Way Quantum Computer. Phys. Rev. Lett 86, 5188 (2001).

10. Sen(De), A. \& Sen, U. Quantum Advantage in Communication Networks. Phys. News 40, 17-32 (2010).

11. Huber, M., Mintert, F., Gabriel, A. \& Hiesmayr, B. C. Detection of High-Dimensional Genuine Multipartite Entanglement of Mixed States. Phys. Rev. Lett. 104, 210501 (2010).

12. Huber, M. \& Sengupta, R. Witnessing Genuine Multipartite Entanglement with Positive Maps. Phys. Rev. Lett. 113, 100501 (2014).

13. Vicente, J. I. d. \& Huber, M. Multipartite entanglement detection from correlation tensors. Phys. Rev. A 84, 062306 (2011).

14. Wu, J. Y., Kampermann, H., Bruß, D., Klöckl, C. \& Huber, M. Determining lower bounds on a measure of multipartite entanglement from few local observables. Phys. Rev. A 86, 022319 (2012).

15. Huber, M., Perarnau-Llobet, M. \& Vicente, J. Ide Entropy vector formalism and the structure of multidimensional entanglement in multipartite systems. Phys. Rev. A 88, 042328 (2013).

16. Sperling, J. \& Vogel, W. Multipartite Entanglement Witnesses. Phys. Rev. Lett. 111, 110503 (2013).

17. Eltschka, C. \& Siewert, J. Entanglement of Three-Qubit Greenberger-Horne-ZeilingerCSymmetric States. Phys. Rev. Lett. 108, 020502 (2012).

18. Klöckl, C. \& Huber, M. Characterizing multipartite entanglement without shared reference frames. Phys. Rev. A 91, 042339 (2015).

19. Markiewicz, M., Laskowski, W., Paterek, T. \& Żukowski, M. Phys. Rev. A 87, 034301 (2013).

20. Ma, Z. H. et al. Measure of genuine multipartite entanglement with computable lower bounds. Phys. Rev. A 83, 062325 (2011).

21. Chen, Z. H., Ma, Z. H., Chen, J. L. \& Severini, S. Improved lower bounds on genuine-multipartite-entanglement concurrence. Phys. Rev. A 85, 062320 (2012).

22. Hong, Y., Gao, T. \& Yan, F. L. Measure of multipartite entanglement with computable lower bounds. Phys. Rev. A 86, 062323 (2012).

23. Gao, T., Yan, F. L. \& van Enk, S. J. Permutationally Invariant Part of a Density Matrix and Nonseparability of N-Qubit States. Phys. Rev. Lett. 112, 180501 (2014).

24. Bancal, J. D., Gisin, N., Liang, Y. C. \& Pironio, S. Device-Independent Witnesses of Genuine Multipartite Entanglement. Phys. Rev. Lett. 106, 250404 (2011).

25. Eltschka, C. \& Siewert, J. Quantifying entanglement resources. J. Phys. A: Math.Theor. 47, 424005 (2014).

26. Jungnitsch, B., Moroder, T. \& Gühne, O. Taming Multiparticle Entanglement. Phys. Rev. Lett. 106, 190502 (2011).

27. Lancien, C., Gühne, O., Sengupta, R. \& Huber, M. Relaxations of separability in multipartite systems: Semidefinite programs, witnesses and volumes. J. Phys. A: Math. Theor. 48, 505302 (2015).

28. Peres, A. Separability Criterion for Density Matrices. Phys. Rev. Lett. 77, 1413 (1996).

29. Sanpera, A., Tarrach, R. \& Vidal, G. Local description of quantum inseparability. Phys. Rev. A 58, 826 (1998).

30. Rudolph, O. On the cross norm criterion for separability. J. Phys. A: Math. Theor. 36, 5825 (2003).

31. Chen, K. \& Wu, L. A. A matrix realignment method for recognizing entanglement. Quantum Inf. Comput. 3, 193C202 (2003).

32. Chen, K., Albeverio, S. \& Fei, S.-M. Concurrence of Arbitrary Dimensional Bipartite Quantum States. Phys. Rev. Lett. 95, 040504 (2005).

33. Zhang, T. G., Zhao, M. J., Li, M., Fei, S. M. \& Li-Jost, X. Q. Criterion of Local Unitary Equivalence for Multipartite States. Phys. Rev. A 88, 042304 (2013). 


\title{
Acknowledgements
}

This work is supported by the NSFC No. 11775306, No. 11675113, No. 11701568; the Fundamental Research Funds for the Central Universities Grants No. 15CX05062A, No. 16CX02049A, and 17CX02033A; the Shandong Provincial Natural Science Foundation No.ZR2016AQ06,ZR2017BA019; Qingdao applied basic research program No. 15-9-1-103-jch, and a project sponsored by SRF for ROCS, SEM.

\section{Author Contributions}

M. Li and J. Wang wrote the main manuscript text. All authors reviewed the manuscript.

\section{Additional Information}

Competing Interests: The authors declare that they have no competing interests.

Publisher's note: Springer Nature remains neutral with regard to jurisdictional claims in published maps and institutional affiliations.

\begin{abstract}
(c) (i) Open Access This article is licensed under a Creative Commons Attribution 4.0 International License, which permits use, sharing, adaptation, distribution and reproduction in any medium or format, as long as you give appropriate credit to the original author(s) and the source, provide a link to the Creative Commons license, and indicate if changes were made. The images or other third party material in this article are included in the article's Creative Commons license, unless indicated otherwise in a credit line to the material. If material is not included in the article's Creative Commons license and your intended use is not permitted by statutory regulation or exceeds the permitted use, you will need to obtain permission directly from the copyright holder. To view a copy of this license, visit http://creativecommons.org/licenses/by/4.0/.
\end{abstract}

(C) The Author(s) 2017 